\title{
Novel Electroplating Technique for Selective Thick-Metal Structure Formation Utilizing a Thin Photoresist
}

\author{
Dong Geon Jung, Seong Ki Min ${ }^{1}$ and Seong Ho Kong* \\ Graduate School of Electronics Engineering, Kyungpook National University, Daegu, Korea \\ ${ }^{1}$ Agency for Defense Development, Yuseong P. O. Box 35, Daejeon, Korea
}

(Received July 10, 2014; accepted December 15, 2014)

Key words: electroplating, three-dimensional structure, thick metal structure

Great effort has been exerted by many researchers on a selective forming technique for a thick metal using an electroplating process for realizing various three-dimenstional (3D) structures. To fabricate such thick 3D metal structures, photolithography using a thick photoresist (PR) is essential for forming an electroplating mold. This PR mold is filled with a thick metal by the electroplating process. However, the process using a thick PR has many problems such as high cost, complex fabrication and difficulty in PR removal. To avoid these problems, we propose a new method of producing a $3 \mathrm{D}$ thick metal structure. A deeply etched Si mold is first prepared and the mold is then patterned with a thin PR for selective metal filling in deeply etched Si trenches by electroplating. During the thin PR patterning, exposure and development processes are repeatedly conducted several times to remove the PR residue possibly remaining in the deeply etched trenches. As a result, a thin PR is perfectly patterned without residues in the trenches and thick metal structures are selectively formed in deeply etched trenches by the electroplating process.

\section{Introduction}

Recently, the demands for low-cost and high-throughput selective thick-metal forming techniques $(>1 \mu \mathrm{m})$ utilizing an electroplating process have been increased in various fields that require three-dimensional (3D) metal structures such as a throughsilicon via (TSV) and a micro-electromechanical systems (MEMS)-based miniaturized power generator. In such a selective thick metal forming process by electroplating, a thick photoresist (PR) has still been extensively used as an electroplating mold by many researchers. ${ }^{(1-8)}$ In this case, the thickness of the formed metal is limited by that

*Corresponding author: e-mail: shkong@ee.knu.ac.kr 
of the PR mold such that a thick PR should be used for forming thick-metal structures. A thick PR commonly used for this purpose has a longer fabrication time, difficulty in removing the PR residue, and a comparatively high cost. In addition to these problems, the PR mold must always be thicker than the metal structure to be selectively filled. However, the typical height of the PR mold fabricated with the thick PR used mainly in the electroplating process is only about $50-100 \mu \mathrm{m}$. Thus, it is difficult to form a metal structure thicker than $100 \mu \mathrm{m}$ by utilizing only the PR mold.

In this paper, we report the fabrication of a deeply etched Si mold with a thin PR without using a thick PR in order to solve the problems mentioned above. When a thick $\mathrm{PR}$ is used on the Si mold with the deeply etched trenches to complete the Si mold with the PR, some bubbles are generated or trapped inside the trenches, as shown in Fig. 1(b). Consequently, additional vacuum equipment must be utilized to remove the bulbbles from the coated PR. In contrast, no bubble was found when a thin PR was used owing to its lower viscosity than a thick PR. In addition, it is essential to minimize the distortion of the mold in conventional electroplating techniques since the shape of the electroplated material is fully determined by the PR mold itself. However, as the thickness of the PR mold increases, it is not easy to precisely control the shape of the mold using a standard UV source. When compared with that for thin PR films, the lithography for thick PR films brings a new extra set of challenges for process optimization. The bulk absorption effect of a thick PR reduces the effective UV dose at the bottom and this impact is further enhanced by the PR development process, resulting in sloped profiles of the PR mold. ${ }^{(12)}$ Therefore, sometimes a thick PR mold is realized by multistep lithography to prevent the formation of sloped mold profiles. We propose this novel method utilizing a deeply etched Si mold with a thin PR in order to realize thick 3D copper $(\mathrm{Cu})$ structures by the electroplating process. The use of the deeply etched Si mold facilitates the thick-metal formation with a thin PR instead of a thick one. By using a thin PR, the fabrication time could be reduced owing to its simple process procedure and the final mold could be completed with no bubbles in the deeply etched trenches and the distortion of the high-aspect-ratio mold could be minimized. In addition, thin PRs are less costly than thick PRs such as SU-8 and JSR. ${ }^{(9,10)}$ They can also be easily removed using common chemical removers, such as sulfuric acid/hydrogen peroxide mixture (SPM) and acetone. ${ }^{(11)}$

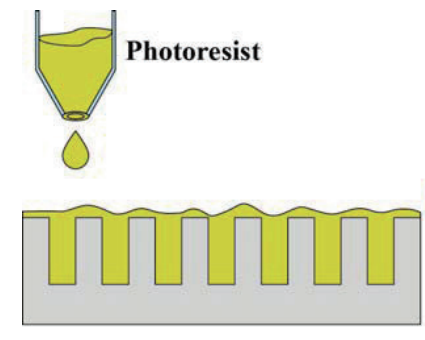

(a)

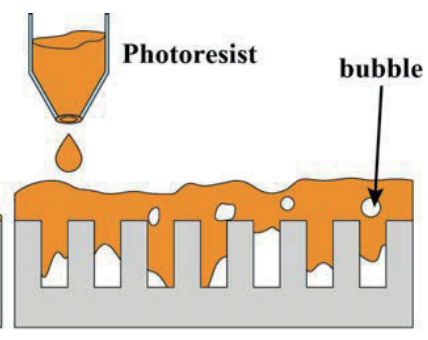

(b)

Fig. 1. (Color online) Spin coating with thick and thin photoresists on deeply etched Si substrate: (a) absence of bubble formation in thin photoresist and (b) bubble formation in thick photoresist. 


\section{Materials and Methods}

A technique for forming thick 3D metal structures by an electroplating process utilizing a deeply etched Si mold with a thin PR has been devised. The thickness of the formed metal is mainly determined by the height of the fabricated Si mold. A positive thin PR (DPR-i5500, Dongjin Semichem) was used as the patterning material. Generally, thin PRs have lower viscosities than thick PRs. Figure 2 shows the fabrication process used to create the Si mold with the deeply etched trenches for the electroplating process. A p-type Si wafer with a thickness of $600 \mu \mathrm{m}$ was used as the substrate and an oxide layer was patterned on the substrate as the mask layer for deep reactive ion etching (DRIE). The depth of the fabricated Si mold through DRIE was firmly controlled considering the intended final metal thickness. After that, oxide and titanium (Ti) layers were deposited as the insulator and seed layers, respectively. Photolithography was then performed in order to form windows for selective metal plating. To formulate a patterned thin PR on the substrate with the deeply etched trenches, we modified the photolithography process,

(a) Si wafer 6 inches in diameter

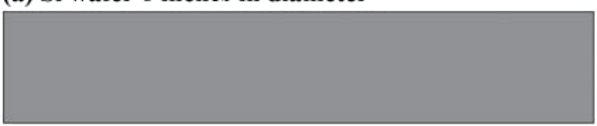

(b) Wet oxidation

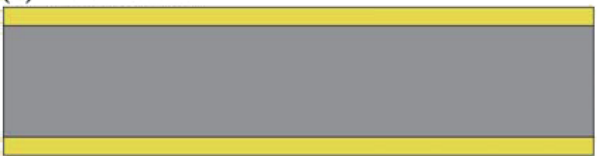

(c) PR patterning

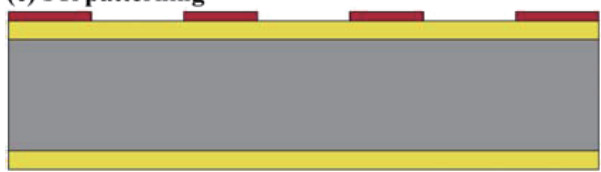

(d) Oxide layer wet etching

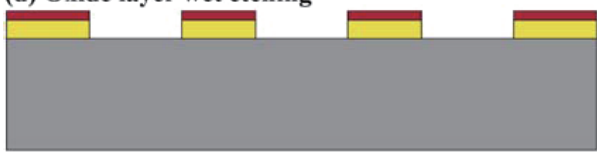

(e) Silicon deep etching

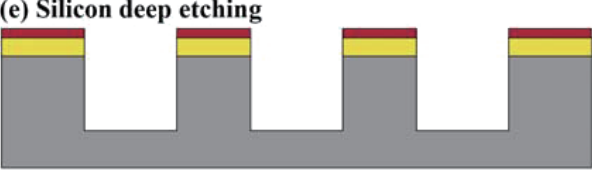

(f) PR and oxide layer removal (g) Deposited $\mathrm{SiO}_{2}$ as insulation layer sputtered $\mathrm{Ti}$ as seed layer

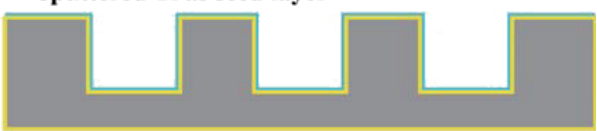

(h) Thin PR patterning

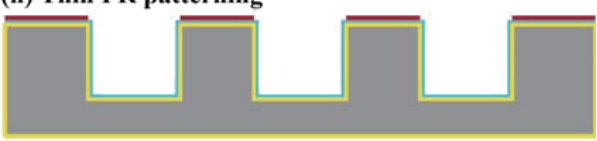

(i) Cu electroplating

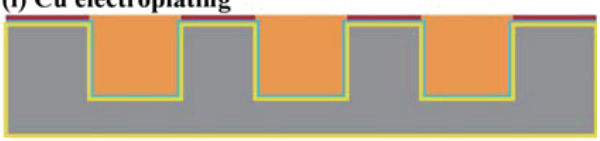

(j) PR removal

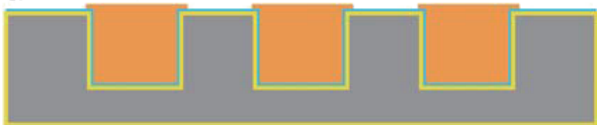

(k) Chemical mechanical polishing

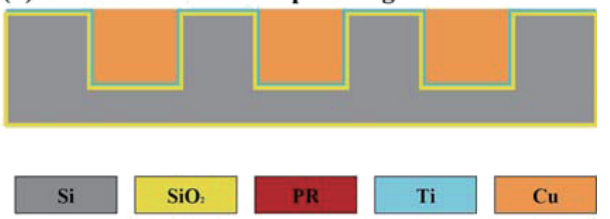

Fig. 2. (Color online) Fabrication process utilizing the proposed novel technique. 
as shown in Fig. 3. Because the thin PR could not be completely removed, especially from the corners of the trenches, by a single step consisting of exposure and development processes, the spin-coated PR was exposed to UV light (i-line spectrum; mercury lamp) and developed by a DPD-200 developer repeatedly. The step-by-step conditions for spin coating and development with the thin PR are shown in Tables 1 and 2, respectively. To pattern the thin PR on deep-trench structures with perfection, we repeated the exposure and development processes four times. The electroplating process was then performed to form thick-metal structures in the deeply etched trenches with the patterned PR. Figure 4 shows the electroplating equipment that we used during the fabrication process. The mixing ratio of the electroplating solution is specified in Table 3.

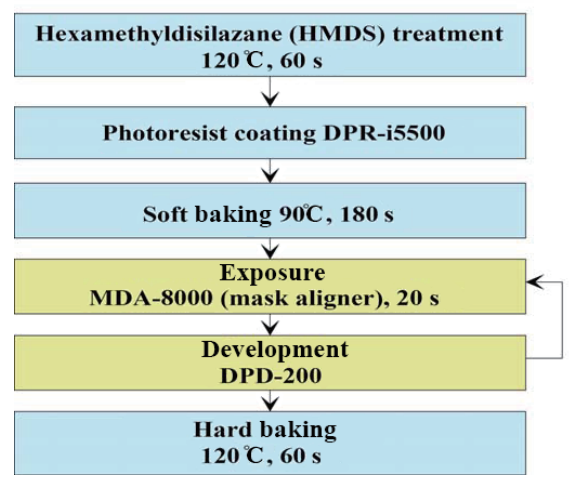

Fig. 3. (Color online) Fabrication sequence of the proposed photolithography process.

Table 1

Spin coating conditions with thin PR.

\begin{tabular}{lrcc}
\hline Step & RPM & Spin time (s) & Accel. time (s) \\
\hline 1 & 500 & 5.0 & 1.0 \\
2 & 1000 & 5.0 & 1.0 \\
3 & 500 & 5.0 & 1.0 \\
\hline
\end{tabular}

Table 2

Development conditions.

\begin{tabular}{lrccc}
\hline Step & RPM & Spin time (s) & Accel. time (s) & Action \\
\hline 1 & 1200 & 5.0 & 0.2 & \\
2 & 2000 & 7.0 & 0.5 & DI water rinse \\
3 & 1500 & 5.0 & 0.2 & \\
\hline 4 & 50 & 2.0 & 3.0 & Developer supply \\
\hline 5 & 0 & 120.0 & 0.0 & Stand by \\
\hline 6 & 2500 & 10.0 & 0.5 & \multirow{2}{*}{ DI water rinse } \\
7 & 2000 & 15.0 & 0.5 & Dry \\
\hline 8 & 3000 & 10 & 0.5 & \\
\hline
\end{tabular}




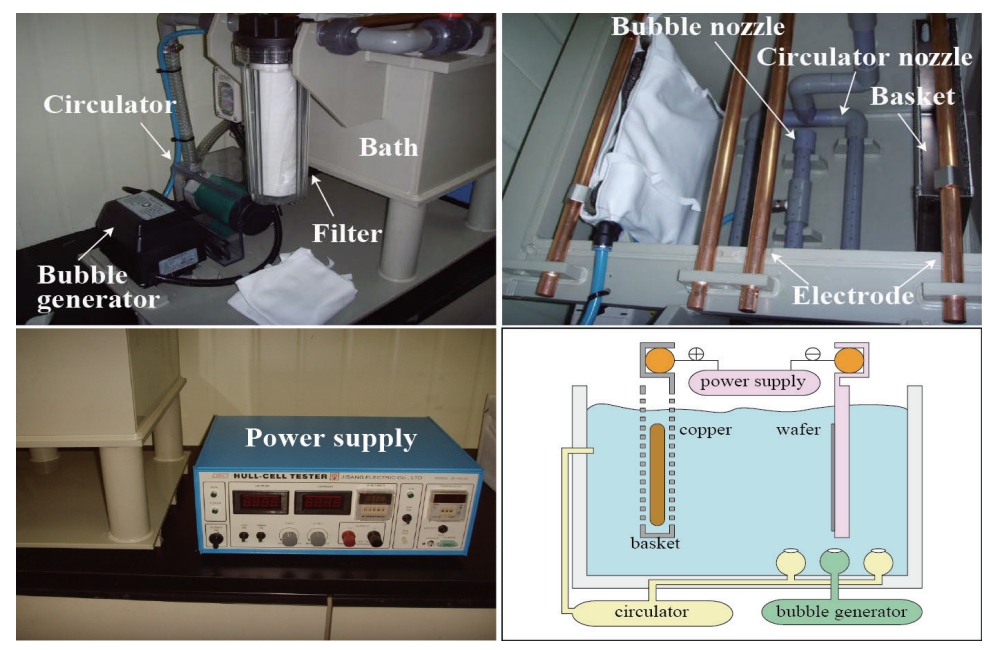

Fig. 4. (Color online) Electroplating equipment.

Table 3

Mixing ratio of electroplating solution.

\begin{tabular}{lc}
\hline For 1 liter of mixed solution & $\mathrm{ml} / \mathrm{L}$ \\
\hline DI water & 85 \\
$\mathrm{CuSO}_{4} \cdot 5 \mathrm{H}_{2} \mathrm{O}$ & 1 \\
$\mathrm{H}_{2} \mathrm{SO}_{4}$ & 34 \\
Cupracid Ultra & 10 \\
Cupracid Ultra Prat A & 0.5 \\
Cupracid Ultra Prat B & 0.5 \\
$\mathrm{Cl}$ & $100 \mathrm{ppm}$ \\
$\mathrm{HCl}$ & 0.0264 \\
\hline
\end{tabular}

\section{Results and Discussion}

Through the developed novel technique, a thin PR was patterned completely without any residue in the trenches formed on the Si mold with a height of $80 \mu \mathrm{m}$, as shown in Fig. 5. When exposure and development processes were performed in a single step, there were residues of the patterned PR in the trenches. When exposure and development processes were performed repeatedly up to 4 times, the residues of the patterned PR in the trenches were completely removed. The patterned PR on the Si mold functions as a mask layer that prevents metal electroplating on top of the deeply etched trenches. Therefore, a selective formation of a thick $\mathrm{Cu}$ film on the Si mold with the deeply etched trenches could be induced. 
The electroplating of $\mathrm{Cu}$ has been conducted under different electrical currents and processing times. Normally, the electrical conditions for the electroplating process are essential for determining the plating quality. In particular, the current density (the current divided by the surface area of the piece) plays an important role in metal plating in determining the quality of a metal. If the current is very high, the plating speed increases, but a porous and coarse plating is achieved. An electroplated metal is deposited as a result of the electric field set up in the plating bath so that the current density distribution also affects the quality of the plated metal, particularly its uniformity. A nonuniform current density distribution at the edge of a trench, especially under highcurrent condition, aggravates the nonuniformity of the plated metal. Therefore, we used an electroplating process in this study with a low current density but for a longer processing time to achieve a void-free and uniform $\mathrm{Cu}$ electroplating in the deeply etched trench structures.

Figure 6 shows the results of $\mathrm{Cu}$ electroplating under different conditions. The process with a low electrical current for a longer processing time gave a superior electroplating result in the deeply etched trench structures. Figure 7 shows that $\mathrm{Cu}$ with a thickness of about $80 \mu \mathrm{m}$ is formed selectively in the Si mold utilizing the proposed technique.
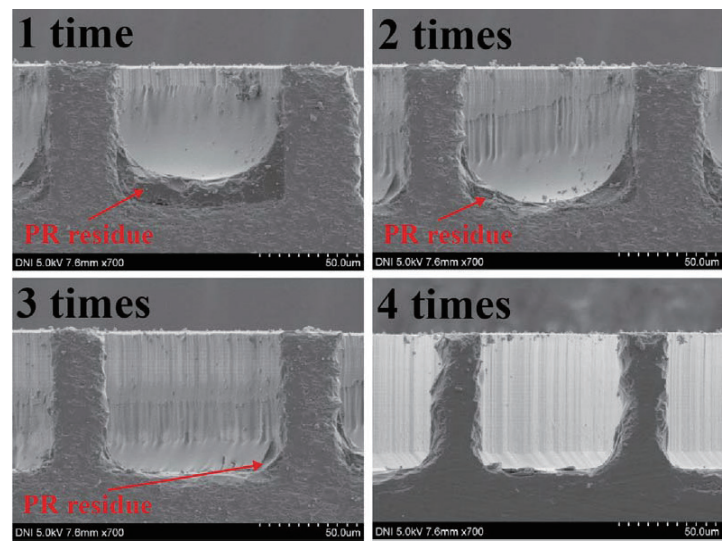

Fig. 5. (Color online) SEM images of the patterned thin PR in trench structure as function of process repetition.

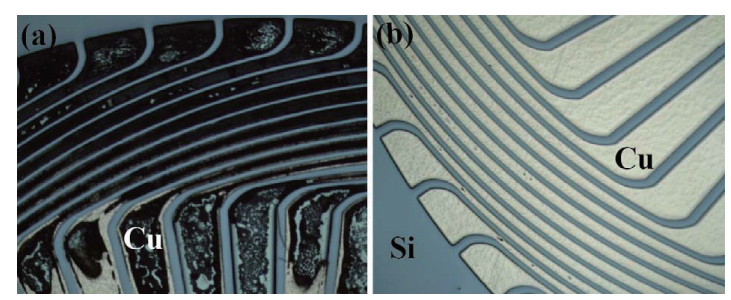

Fig. 6. (Color online) Electroplated $\mathrm{Cu}$ under different plating conditions: (a) $3 \mathrm{~A}$ for $3 \mathrm{~h}$ and (b) 0.8 A for $10 \mathrm{~h}$. 


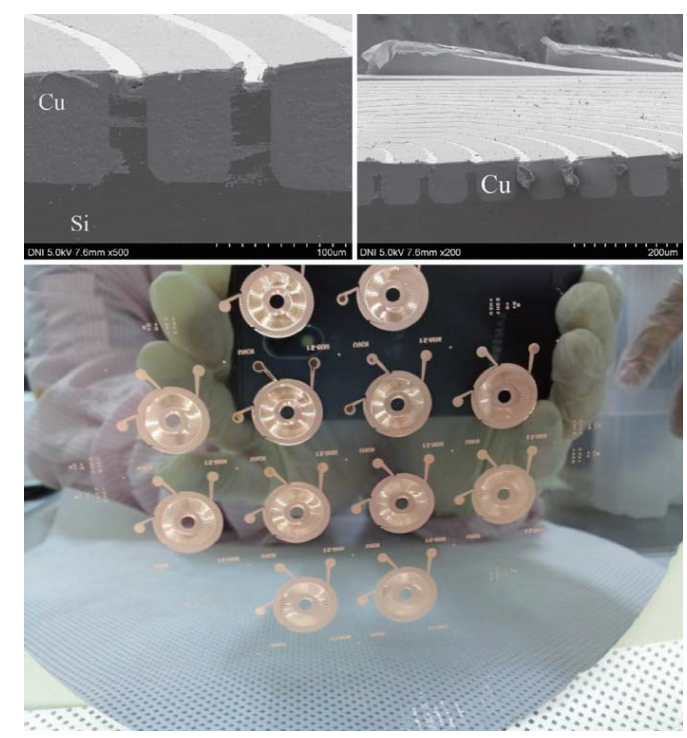

Fig. 7. (Color online) SEM and optical images of the fabricated Si trench structure with the proposed photolithography and electroplating processes.

\section{Conclusions}

In this study, we demonstrated a novel electroplating technique for selective thickmetal structure formation utilizing a deeply etched Si mold, and the repeated exposure and development processes with a thin PR. To confirm the feasibility of the proposed technique to form a thick-metal structure on the Si mold, we mainly performed DRIE, photolithography with a thin PR and an electroplating process. The fabricated Si mold was used for forming a thick metal without a thick PR. The depth of deeply etched trenches on the Si mold determines the thickness of the electroplated metal. In particular, we used a thin PR to overcome the issues accompanying the use of a thick PR such as high cost, complex fabrication procedure and difficulty in PR removal. Repeated exposure and development processes were applied to photolithography with a thin PR in order to completely remove the residues of the patterned thin PR in the deeply etched trenches on the Si mold. We confirmed that the proposed novel technique could facilitate selective thick-metal formation by electroplating.

In summary, the main advantages of the proposed technique are as follows: (1) it enables the formation of a metal thicker than $100 \mu \mathrm{m}$ possible without a thick PR; (2) the repetitive UV exposure and development processes could entirely remove residues of PR, even in the deeply etched trenches; and (3) the proposed technique is less expensive than the electroplating process utilizing a thick PR. Therefore, the proposed novel electroplating technique with a thin PR utilizing a deeply etched Si mold could be extensively applied in various fields such as those involving TSVs, 3D micro/ nanostructures and devices. 


\section{Acknowledgements}

This research was supported by the National Research Foundation of Korea Grant funded by the Korean Government (2008-0062617) and financially supported by a grant to the MEMS Research Center for National Defense from DAPA/ADD.

\section{References}

1 W. W. Flack and H. A. Nguyen: SPIE (2003) 151.

2 W. W. Flack and H. A. Nguyen: SPIE (2005) 103.

3 E. Kukharenka, M. M. Farooqui, L. Grigore, M. Kraft and N. Hollinshead: J. Micromech. Microeng. 13 (2003) S67.

4 J. J. Maes, A. G. van Nie and G. B. A. Hut: Microelectron. Reliab. 17 (1978) 325.

5 J. K. Luo, D. P. Chu, A. J. Flewitt, S. M. Spearing, N. A. Fleck and W. I. Milne: J. Electrochem. Soc. 152 (2005) C36.

6 W. W, Flack, H. A. Nguyen and J. Buchanan: SPIE (2004) 164.

7 T. Deng, F. Arias, R. F. Ismagllov, P. J. A. Kenis and G. M. Whitesides: Anal. Chem. 72 (2000) 645.

8 B. Loechel: J. Micromech. Microeng. 10 (2000) 108.

9 C. Chunwei, P. Robert, Ng. Edward, L. Sam, M. Stephen, P. Georg and B. Rozalia: SPIE 923 (2008) 69233E.

10 N. Basak, G. L. Harris, J. Griffin and K. D. Wise: Micro\&Nano Lett. 2 (2007) 115.

11 P. M. Dentinger, W. M. Clift and S. H. Goods: Microelecron. Eng. 61 (2002) 993.

12 J. Gau: SPIE 1088 (1989) 504. 